\title{
COMMENTS ON THE REFLECTION OF ELECTRONS FROM STANDING LIGHT WAVES
}

\author{
L.S. BARTELL \\ Department of Chemistry, The University of Michigan, Ann Arbor, Michigan 48104, USA
}

Received 10 June 1968

\begin{abstract}
Certain discrepancies in a recent paper purporting to describe the successful observation of an effect predicted by Kapitza and Dirac are discussed. It is concluded that the claims for success are inadequately supported by the evidence.
\end{abstract}

Several years ago this journal published a brief report [1] of an alleged observation of the Kapitza-Dirac effect [2], the reflection of electrons by standing light waves. Recently, a more detailed account of the experimental work was presented in which "the first successful observation" of the Kapitza-Dirac effect was claimed [3]. We feel it appropriate to criticize the experimental accounts on the following grounds: a) the evidence adduced falls substantially short of that usually considered to constitute an acceptable demonstration of a new effect, and b) the results presented do not appear to be consistent with the Kapitza-Dirac theory, contrary to assertions by the author [3]. In addition, certain crucial experimental details are omitted or are described too incompletely to permit a proper evaluation of some extraordinary claims for the electron optical performance.

Returning to point (a), above, we note the ease with which spurious, laser-induced signals of an incompletely understood nature can be obtained in studies of the Kapitza-Dirac effect with high intensity light waves [4-6]. Therefore, it is essential to submit the observed signals to rigorous diagnostic tests. Even in the absence of fully characterized experimental conditions, certain criteria can be for mulated against which experiments should be tested if observations are to be accepted as bona fide [6]: (I) signals should occur simultaneously with the laser pulses (delayed or prolonged responses not infrequently occur); (II) signals should be observable at total deflection angles within a focussed electron beam width of the expected Bragg law value; they should vanish at smaller and at larger values; (III) signals should vanish unless the angle of incidence of the electrons is consistent with Bragg's law; and IV the observed electron reflection probability should be in reasonable conformity with theory.

It is instructive to apply the above criteria to the results in question $[1,3]$, which fall into two categories: type $A$ (slow detector) responses which were not subjected to criterion $I$, and type $B$ (fast detector) responses which meet criterion I well. Criterion I is not very discriminating if the electron beam passes close to the laser reflection [6]. Criterion II is vital in the discrimination against noise because the highly directed nature of the Kapitza-Dirac recoils is the most characteristic aspect of the phenomenon (a stimulated Compton effect). Indeed, if criterion II were met unequivocally with an extremely wellfocussed electron beam (an order of magnitude finer than the Bragg angle, say), then one would have considerable confidence in the experiment even if the other criteria were only cursorily tested. Inasmuch as the detectors in the experiments in question were not designed to give any measure of the distribution of scattered intensity over scattering angles, no information on the scattering angle was established. Criterion III, to test the Bragg restriction on angle of incidence, would be valuable in a test analogous to a single crystal diffraction experiment. In studies with neodymium laser radiation $[1,3]$, however, the large wave length inhomogeneity gives a distribution of tilted effective Bragg planes more characteristic of a powder.

Contrary to the published conclusions [3], neither the type A nor type $B$ results appear to be of a reasonable order of magnitude. As for type A signals, even granting that the intensity of the laser's normal burst mode spikes reached the large value of 100 megawatt $/ \mathrm{cm}^{2}$ required to 
deflect half the electrons, and accepting the even more unlikely condition that the electrons were deflected in one direction only by the distributed planes, it is difficult to see how an average of half of the electrons could have been deflected by light waves during the $500 \mu \mathrm{sec}$ periods illus trated in fig. 4a [3] when the laser was firing only $1 / 20$ of the time! On the other hand, spurious deflections of the type noted by other authors $[4,6]$ could account for large, persisting signals.

The type B signals are of a different nature, for they are observed only when the laser spikes appear. However, the behavior they exhibit would be highly improbable for Kapitza-Dirac signals in view of the large $\Delta \lambda$ associated with the laser used. "Standing waves" suitable for reflecting electrons may be generated when an incident light wave of one frequency combines with a reflected wave of another frequency. Since these waves are stationary only in the moving reference frame in which the component frequencies are made equal by Doppler shifts, their nodal planes are tilted relative to the incoming electron beam. Tilt angles correspond to $\pm c \Delta \lambda / 2 \lambda v$ [5], or nearly 1 radian for characteristic neodymium laser outputs with $\Delta \lambda \approx 100 \AA$. Accordingly, because the electron beam encounters a random angular distribution of tilted Bragg planes, it is to be anticipated that electrons would reflect to the right (positive diffraction order) as often as they would reflect to the left (negative diffraction order). This would give the difference $\left(I_{R}-I_{L}\right)$ between right and left scattered currents an expectation value of zero. The experiments in question $[1,3]$ were designed to measure the difference $\left(I_{R}-I_{L}\right)$ rather than the individual $I_{R}$ or $I_{\mathrm{L}}$ currents. It was asserted that the observed signal was of the magnitude to be expected for reflection in one direction. Consequently, the measured difference appears to exceed the theoretically expected difference by orders of magnitude.

It might be argued that a given laser pulse could generate Bragg planes so oriented that the incident electron beam would reflect in only one direction. Since the orientation of such Bragg planes would be unfavorable for reflection in the other direction by, at most, twice the Bragg angle $\left(\sim 3 \times 10^{-4}\right.$ radians), this argument would necessitate the postulation of an extremely sharp cut-off in the wave length and angular distributions, together with an exceedingly delicate align ment. It seems inconceivable that succeeding laser pulses would all retain this special distribution and accidental alignment, yet the type $B$ traces all show the "reflected electrons" going in the same direction with substantially equal intensity during several irregular series of laser pulses [3]:

For the above reasons it is not unreasonable to conclude that the reported signals represent noise. Several arguments were advanced [3] which purported to rule out noise as a source of the signals. It was suggested that noise would contribute equally in the right- and left-hand detectors, and, hence, would cancel. This disregards the asymmetry of the environment of the electron beam in its passage through the laser cavity. Contrary to allegations, spurious type A signals could arrive with double the modulation frequency if, for example, the detection wires were slightly bowed (microns per centimeter length) or if the spurious deflection field were nonuniform. Furthermore, delocalized surface and/or or space charge effects could not vary as rapidly with distance as asserted. Therefore, the reported diagnoses do not rigorously discriminate against spurious responses.

We are forced to conclude that the reported signals $[1,3]$ are unlikely to represent true Kapitza-Dirac recoils. Quite apart from the evidence against the experimental results, it is inescapable that the most crucial criteria to be satisfied were not even tested. Therefore, it is clear that the author's claim [3] that he 'actually observed" the Kapitza-Dirac effect is premature.

\section{References}

1. H. Schwarz, H.A. Tourtellotte and W. W. Gaertner, Phys. Letters 19 (1965) 202.

2. P. L.Kapitza and P.A.M.Dirac, Proc. Camb. Phil. Soc. 29 (1933) 297.

3. H.Schwarz, A. Physik 204 (1967) 276.

4. H.Pfeiffer, Phys. Letters 26 (1968) 362; L. S. Bartell, H. B. Thompson and R. R. Roskos, Phys, Rev. Letters 14 (1965) 851.

5. L.S. Bartell, J. Appl. Phys. 38 (1967) 1561.

6. L.S. Bartell, R. R. Roskos and H.B. Thompson, Phys. Rev. 166 (1968) 1494. 